\title{
Regolith Excavation Performance of a Screw-propelled Vehicle
}

\author{
Marko Green ${ }^{1}$, Teresa McBryan ${ }^{1}$, Darwin Mick $^{1}$, David Nelson ${ }^{1}$, and Hamid Marvi ${ }^{1}$ \\ ${ }^{1}$ Affiliation not available
}

August 6, 2021

\begin{abstract}
Excavation of regolith is the enabling process for many of the in-situ resource utilization (ISRU) efforts that are being considered to aid in the human exploration of the moon and Mars. Most proposed planetary excavation systems are integrated with a wheeled vehicle, but none yet have used a screw-propelled vehicle which can significantly enhance the excavation performance. Therefore, CASPER, a novel screw-propelled excavation rover is developed and analyzed to determine its effectiveness as a planetary excavator. The excavation rate, power, velocity, cost of transport, and a new parameter, excavation transport rate, are analyzed for various configurations of the vehicle through mobility and excavation tests performed in silica sand. The optimal configuration yielded a $30 \mathrm{~kg} / \mathrm{hr}$ excavation rate and $10.2 \mathrm{~m} / \mathrm{min}$ traverse rate with an overall system mass of $3.4 \mathrm{~kg}$ and power draw of less than $30 \mathrm{~W}$. These results indicate that this architecture shows promise as a planetary excavation because it provides significant excavation capability with low mass and power requirements.

Corresponding author(s) Email: hmarvi@asu.edu
\end{abstract}

\section{Table of Contents}




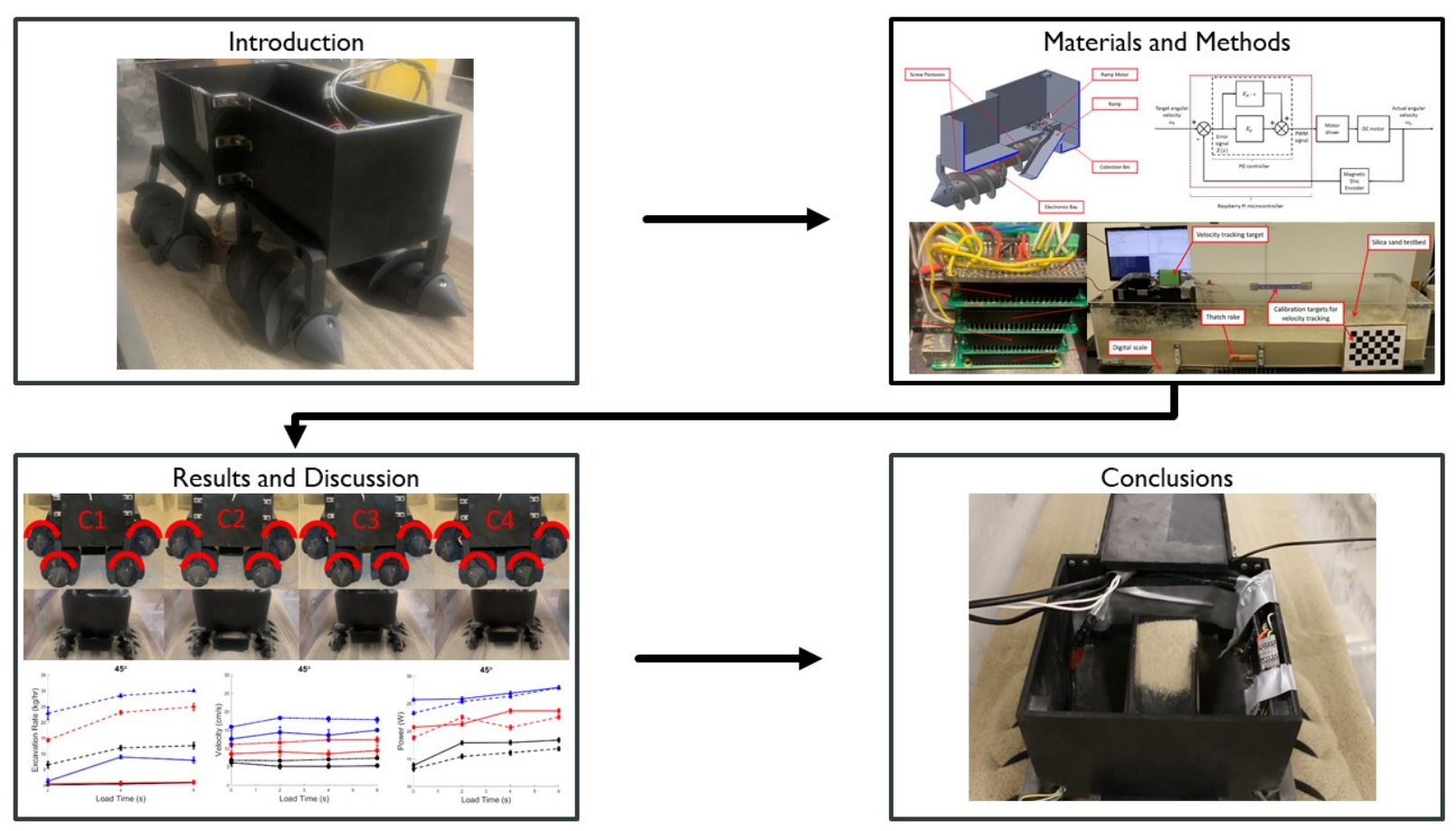

Figure 1: This paper will begin with an introduction which covers the motivations for this research and a review of the relevant scientific literature. It will then transition into a discussion of the materials and methods of the research presented, including design of the CASPER prototype and experimental design. Next, the results of the experiments will be presented and discussed. Finally, the paper will conclude with a summary of the key results, and a discussion of potential avenues for future study.

\section{Introduction}

In situ space excavation is a highly researched and sought after branch of technology as it will help achieve numerous scientific goals and enable a sustained human presence via in-situ resource utilization (ISRU) (Benaroya et al., 2002; Labeaga-Martínez et al., 2017; Cesaretti et al., 2014; Saxena et al., 2019; Li et al., 2018; Zuppero et al., 1997; Blair et al., 2002; Jones et al., 2019; Crawford, 2015; Lavoie and Spudis, 2016; Sanders, 2011; Sanders and Larson, 2012; Spudis and Lavoie, 2011; Mueller and King, 2008). In order to achieve the goals of ISRU, robotic vehicles capable of traversing planetary surfaces and excavating material must be developed. According to the literature (Just et al., 2020b; Skonieczny et al., 2011), excavation systems are either discrete, where one cutting surface makes repeated contact with the surface, or continuous, where multiple cutting surfaces successively make contact with the surface to excavate material. Just et al. (Just et al., 2020b) also indicate that the key parameters that should be used to compare excavation performance are the excavation rate, vehicle traverse rate, total power consumption, and the geotechnical properties of the regolith analogue used. These parameters are indeed critical to characterizing the performance of ISRU excavation systems, however, this paper will examine two additional parameters that provide further insight into system performance: the cost of transport (COT), which is a measure of mobility efficiency and the excavation transport rate (ETR), a new parameter introduced in this work that seeks to combine excavation and mobility performance. In particular, ETR is the rate at which the excavation system collects material, normalized by the craft mass and cost of transport.

The majority of research on ISRU excavation systems has focused primarily on the development of wheel and track based excavation systems. Scarab (Wettergreen et al., 2009, 2010) and RASSOR (Mueller et al., 
2013) are two examples of wheeled excavation platforms, which both employ continuous excavation systems, a drill and bucket wheel, respectively. Conversely, Cratos (Greer et al., 2013; Caruso et al., 2013) utilizes a discrete scraper excavation system. It employs a track based mobility system due to the large tractive forces required by the scraper. Furthermore, studies into the excavation force requirements for space environments have been conducted (Zeng et al., 2007). The large excavation forces required for excavation, coupled with the low weight and traction forces experienced on planetary surfaces imply that the excavation system should minimize the excavation force, and maximize the amount of traction provided by the mobility system in order to enable efficient excavation for ISRU (Wilkinson and DeGennaro, 2007). Continuous systems have typically been found to be more advantageous for low-gravity planetary environments because they require less tractive force than discrete systems (Skonieczny et al., 2016).

One key mobility architecture that has been neglected until now is a counter-rotating Archimedes screw propelled mobility system, which uses rotating pontoons with helical protuberances to react against granular media and propel the vehicle forward. Screwed-Propelled Vehicles (SPVs) have been shown to provide large tractive forces even in reduced gravity environments (Thoesen et al., 2019c, 2018). Additionally, a screw mobility system can be used to augment an excavation system by churning up material prior to being collected, thereby reducing the required force to excavate material. The high tractive force and potential for reducing the force required to excavate material make screw-propelled vehicles highly interesting as a candidate for an ISRU excavation system. On Earth, SPVs have traditionally been used in terrestrial applications in amphibious or otherwise challenging environments (Neumeyer and Jones, 1965; Evans, 2016; Ju et al., 2010) where sand, marshes, clay, or ice are present. These environments prevent vehicles using wheels or tracks from being reliably mobile. However, SPVs are well suited to those terrains because they are mechanically simple and provide a large surface area for traction. Screw generated forces in granular media have been analyzed both experimentally and computationally (Thoesen et al., 2019c, 2018). Furthermore, screw-driven mobility platforms have been tested in a lunar regolith analogue and computationally tested using gravity variant coupled discrete element method and multi-body dynamics simulations (Thoesen et al., 2020a,c,d, 2019b). Results indicate that screw propelled vehicles provide high tractive forces and are capable of mobility across a wide range of gravities. In addition, scaling laws have been developed that relate gravity and craft size to the craft's velocity and power draw (Thoesen et al., 2020a,c, 2019a, 2020d). Building upon this prior body of work, a screw-propelled excavation rover is developed and serves as the test subject for this analysis. This vehicle aims to meet the requirements of in-space excavation by using the screw propulsion system to provide high tractive force and reduce excavation force through churning the granular media and reducing its compaction.

The Counter-rotating Archimedes Screw-Propelled Excavation Rover (CASPER) is a novel platform that combines screw-propelled mobility with a discrete scooper excavation method. CASPER uses four screws to move itself forward while a centrally located ramp excavates material during rover movement. This combination of excavation technique augmented by a screw mobility system has not been investigated before, and will be compared to other techniques using the parameters described above. This initial iteration of CASPER is a prototype which is meant to serve as a proof of concept for a helical mobility system augmenting a discrete scooper excavation system, and is not intended to be used on planetary surfaces for ISRU excavation. Future iterations of the craft would require improved materials for the body, excavation, and mobility systems, in addition to a robust suspension system to be capable of traversing and excavating material from planetary surfaces. The vehicle developed and the analysis methods presented in this paper are new to the field of planetary excavation in the following ways: The CASPER prototype is the first proposed vehicle to use a counter-rotating Archimedes screw mobility system to augment an excavation system of any type. Particularly, the CASPER excavation system is the first discrete scooper to be studied and compared to continuous excavation systems (Skonieczny et al., 2016). In addition, the excavation transport rate is a novel parameter that can be used as a method to compare the combined mobility and excavation performance of ISRU excavators. We will first discuss the design of the CASPER rover and the experimental procedures. Next we will present our experimental data on CASPER mobility and excavation performance. Finally, we will conclude with the major takeaways from this analysis and potential future directions. 


\title{
Materials and Methods
}

\author{
Design of CASPER
}

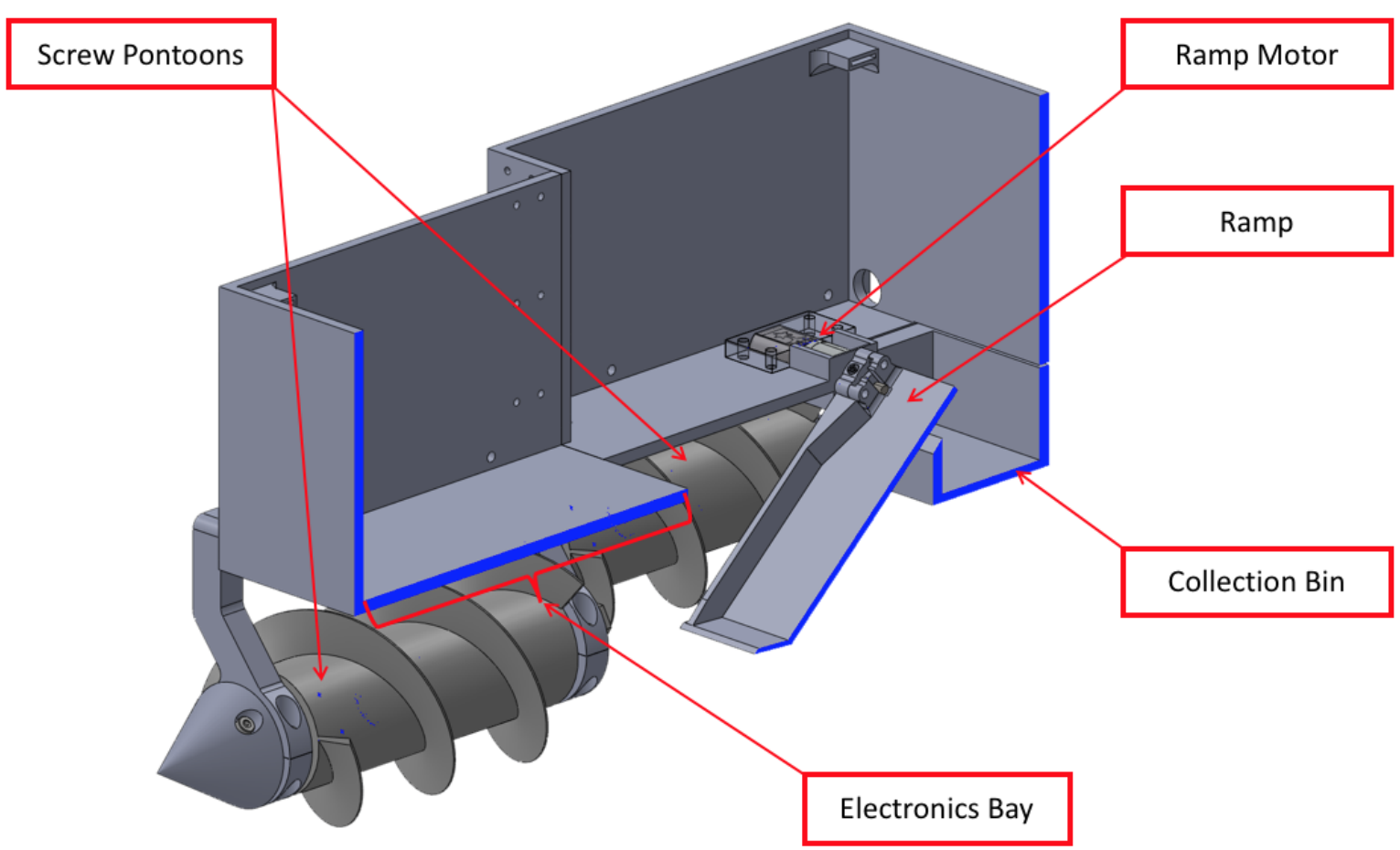

Figure 2: Isometric section view of CASPER with key features labeled.

CASPER is a screw-propelled discrete scooper excavation robot, and is shown in Figure 2 and Figure 3. It is driven by a system of four counter-rotating Archimedes screw pontoons. Each of these pontoons are driven by an internal $12 \mathrm{~V}$ DC motor which is sealed against sand ingress. The pontoons serve primarily as the mobility system, but also play a key role in the excavation system. The excavation system consists of two major components, the pontoons, and the ramp. The pontoons churn up the material and propel the ramp forward, while the actuated ramp collects the material and rotates to deposit the material into the collection bin. The majority of CASPER parts were 3D printed in polyethylene terephthalate glycol (PETG) on an fused deposition modeling (FDM) 3D printer.

A Raspberry Pi was used to control all craft functions and collect experimental data. The screw pontoons were each powered by a 75:1 DC gear motor (Pololu; item \#4846) and the ramp was actuated using a 488:1 DC gear motor (Polulu; item \#3723). The ramp required a very high gear ratio motor because it needed to be able to maintain the desired angle while experiencing very high drag forces from the granular media. Each motor comprised of a motor, gearbox, encoder, current sensor, and a motor controller for speed control. The craft was tethered to and powered by an external power supply providing a constant voltage of $12 \mathrm{~V}$ 


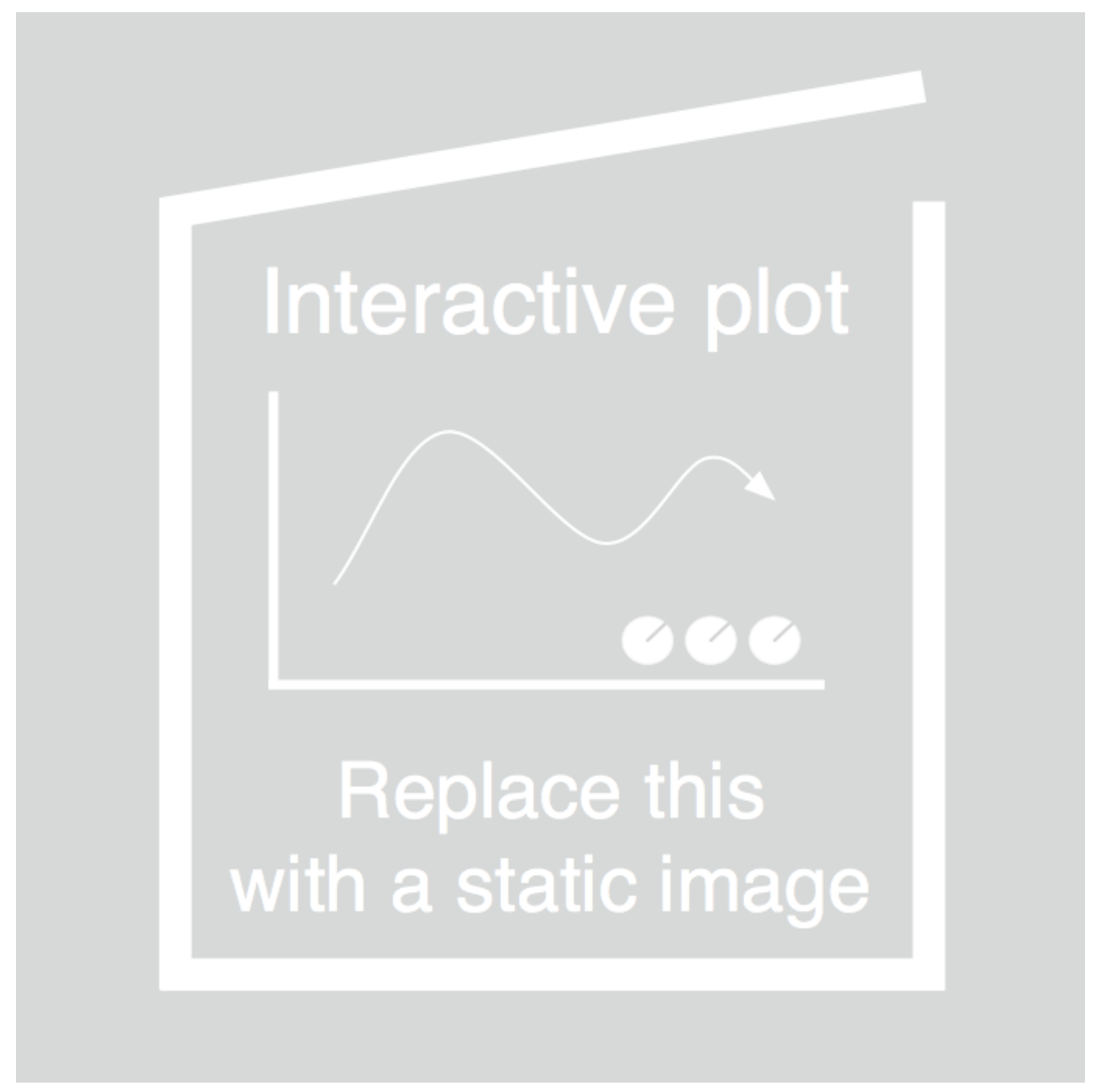

Figure 3: Interactive CASPER CAD Model

to the system. A proportional-derivative (PD) controller was utilized to regulate pontoon speeds to ensure accurate speed control and was tuned such that the craft consistently achieved steady state in approximately $0.9-1.3$ seconds for all screw angular velocity settings. This guaranteed that the craft would be at steady state when the ramp excavation procedure began at 2 seconds after run start.

Four major configurations of CASPER were tested during the course of this research. The parameters of each configuration and their physical layout, rotation directions, and wake images are shown in Figure 4. Each configuration had a unique combination of front pontoon width (narrow or wide), and the direction in which material was churned by the front pontoons (inward or outward). Configurations C1 and C2 (pictured in Figure 4) have pontoon mounts that create a $145 \mathrm{~mm}$ separation between the centers of the front pontoons, while the $\mathrm{C} 3$ and $\mathrm{C} 4$ configurations have a narrow set of pontoon mounts that create $105 \mathrm{~mm}$ of separation. Furthermore, the even configurations $\mathrm{C} 2$ and $\mathrm{C} 4$ have front pontoon handedness and rotation direction opposite of the rear screws, which causes the material to be churned inward by the front pontoons. This "inward churning" creates a wake of loosely compacted material that is elevated with respect to the undisturbed sand, which enables the ramp to easily scoop the material in discrete amounts. Conversely, the odd configurations $\mathrm{C} 1$ and $\mathrm{C} 3$ have identical screw handedness and rotation direction as the rear pontoons, which causes the material to be churned outward and away from the central ramp. For each configuration, the power, velocity, and excavation rate of the craft were investigated over a range of each of the following independent variables:

- Angular Velocity $\omega$ : The rotation rate of each screw pontoon. 


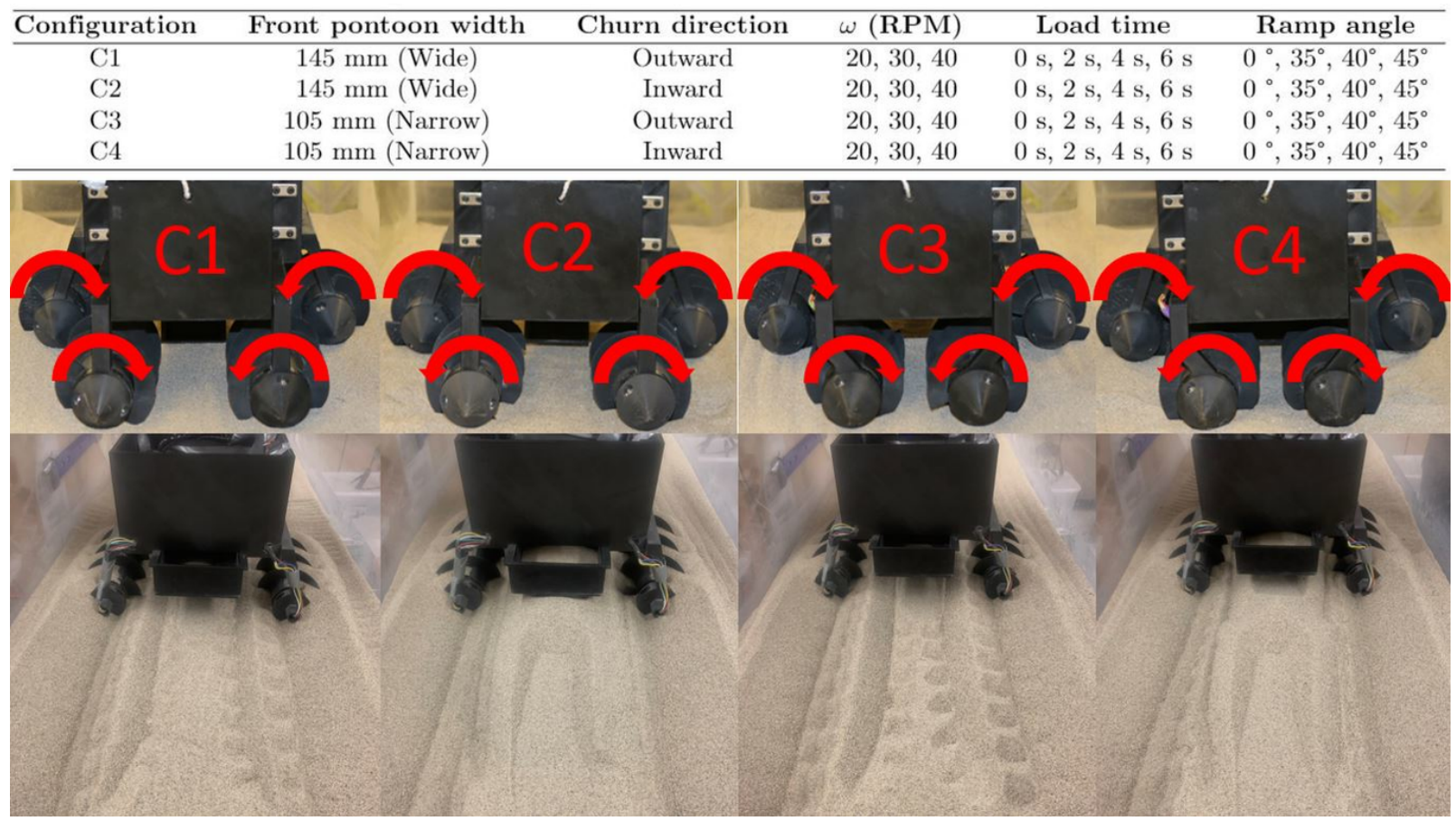

Figure 4: Parameters and front view of each configuration with indicated screw rotation direction and their corresponding wakes.

- Load time: The length of time where the ramp is in the downward fixed position, embedded in the granular media, moving forward, and collecting material.

- Ramp angle: The angle that the ramp surface makes with respect to the floor of the craft body.

It should be noted that the 0 degree ramp angle and 0 second load time cases act as the control group for the mobility power and velocity. This is because during these specific runs, the ramp was disabled in the upright stowed position, so the power and velocity were unaffected by reaction forces due to the ramp excavation.

The purpose of these experiments is to determine what effect, if any, the material churn direction and front pontoon width have upon the power consumption, velocity, and excavation rate of the craft across a wide variety of screw angular velocities, load times, and ramp angles.

\section{Experimental Setup and Procedure}

The experiments were performed in a $150 \mathrm{~cm}$ x $45 \mathrm{~cm}$ x $45 \mathrm{~cm}$ acrylic box (Figure 5) filled with commercially available Quikrete 1962 Eglin silica sand that has a mean particle diameter of 0.8-1.8 mm (Nardelli et al., 2017). Quikrete is a very common granular media to perform mobility experiments in and this is a legacy test bed from past experiments (Thoesen et al., 2020a,c, 2019a, 2020d). Quikrete silica sand was added to the test bed such that there was $20 \mathrm{~cm}$ of depth in order to prevent any wall effects from occurring between the screws and the bottom of the test bed.

Prior to each trial, the sand was reset manually using a modified thatch rake using a strictly consistent procedure. This was done to minimize the variance in measured quantities due to a buildup of plastic deformation in the sand. It has been shown that inconsistent soil conditions cause variability in the interactions of the mobility system and the granular media (Heverly et al., 2013), which increases variance in the measured performance parameters. First, the craft would be removed from the soil. Next, the tines of the thatch rake 


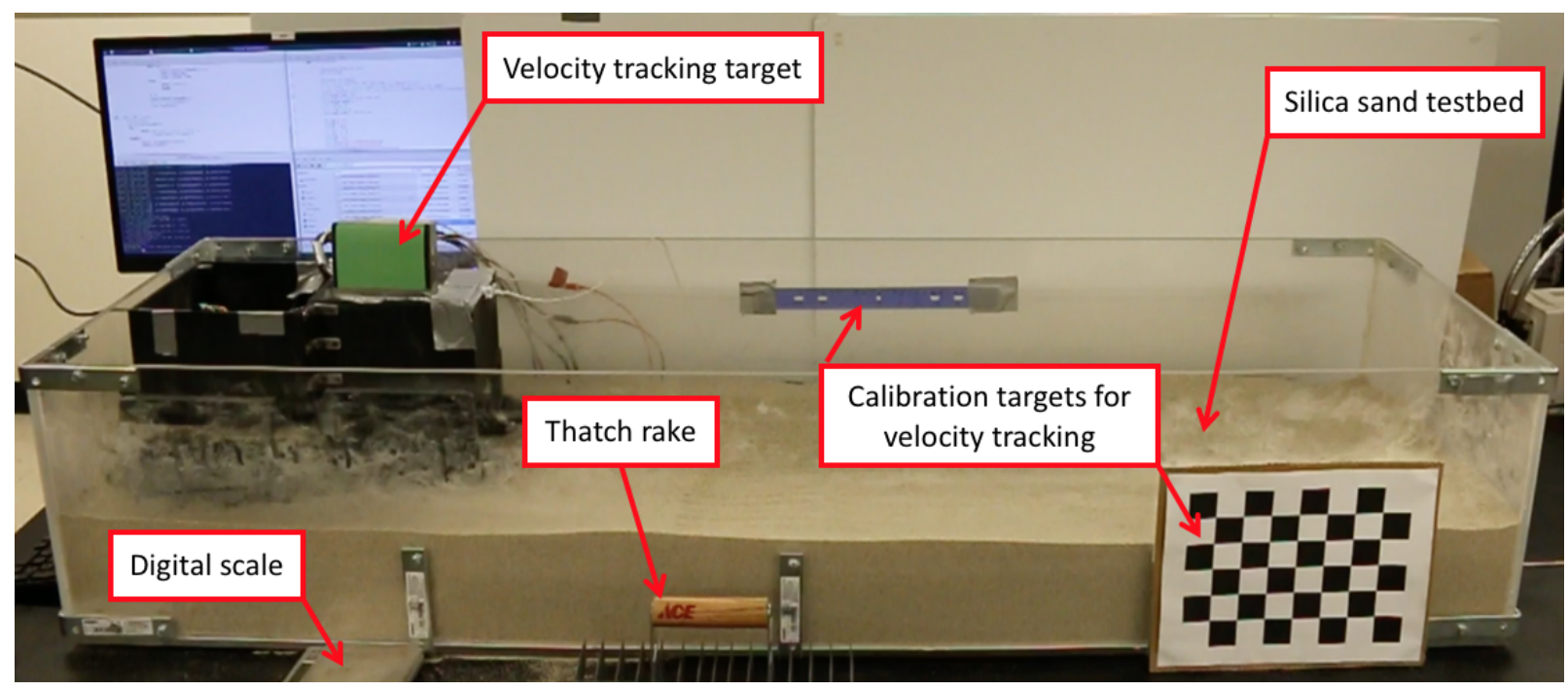

Figure 5: Experimental setup with key equipment labeled.

would be drawn in longitudinal and transverse directions repeatedly until the sand was no longer compacted. Finally, the sand level was checked to make sure that the volume fraction of the sand remained constant between trials.

Once the sand was reset, the craft was replaced at the end of the test bed, and the ramp angle was set using a digital angle gauge and manual inputs to the craft's control system. After this process, the camera was set to record and the control script was launched at the command line to start the run. The craft was then allowed to traverse forwards until the ramp had deposited two discrete scoops into the collection bin, thus concluding the run. Two scoops were chosen because this is the maximum amount of scoops possible in this length of a test track at the highest screw angular velocity of 40 RPM with the longest load time of 6 seconds. Finally, the collection bin was removed and weighed using a digital scale and the resulting value was recorded. After recording the mass, the process was repeated for a multiplicity of five trials per experimental configuration.

The RPM, voltage, and current data vectors for each of the five motors were analyzed in MATLAB. For each trial, the current and voltages of each motor were multiplied together to obtain the electrical power supplied to the DC motors. Next, the time average of the power curves were taken across the temporal range where the craft had reached steady state, as indicated by the RPM versus time curve. The time-average power values for all five trials were recorded, and the ensemble average of these values was taken to determine the average power consumption for that specific set of experimental parameters.

Next, the videos of each run were analyzed using a color tracking MATLAB script. The camera was calibrated by taking several images of the checkerboard, including ones planar to the green color tracking target, and analyzing them in MATLAB to get the camera's intrinsic and extrinsic parameters. This enabled the pixel coordinates to be converted into world coordinates. The script analyzed each video frame by frame, finding the location of the center of the green target and thus, giving distance over time and therefore velocity. The average velocity was then calculated by taking the time-average of the velocity over the duration where the craft was considered in steady state. The values for all five trials were taken and averaged together to get an average velocity for that specific set of experimental parameters. A video exhibiting the operation of the excavation system during the experimental process is shown in Figure ??.

Rich media available at https://www . youtube. com/watch?v=Vgd4sMD8p5Y 


\section{Results and Discussion}

As mentioned in the previous section, the purpose of these experiments was to quantify the effect the material churn direction and front pontoon width have upon the power consumption, velocity, and excavation mass performance of the craft across a wide variety of screw angular velocities, load times, and ramp angles. In the following subsections, the experimental data for the excavation rate, power, velocity, cost of transport, and excavation transport rate are presented and analyzed. It should be noted that the mobility and excavation performance was consistent across all data collection runs and that no drop-off in performance was observed.

\section{Excavation Rate}
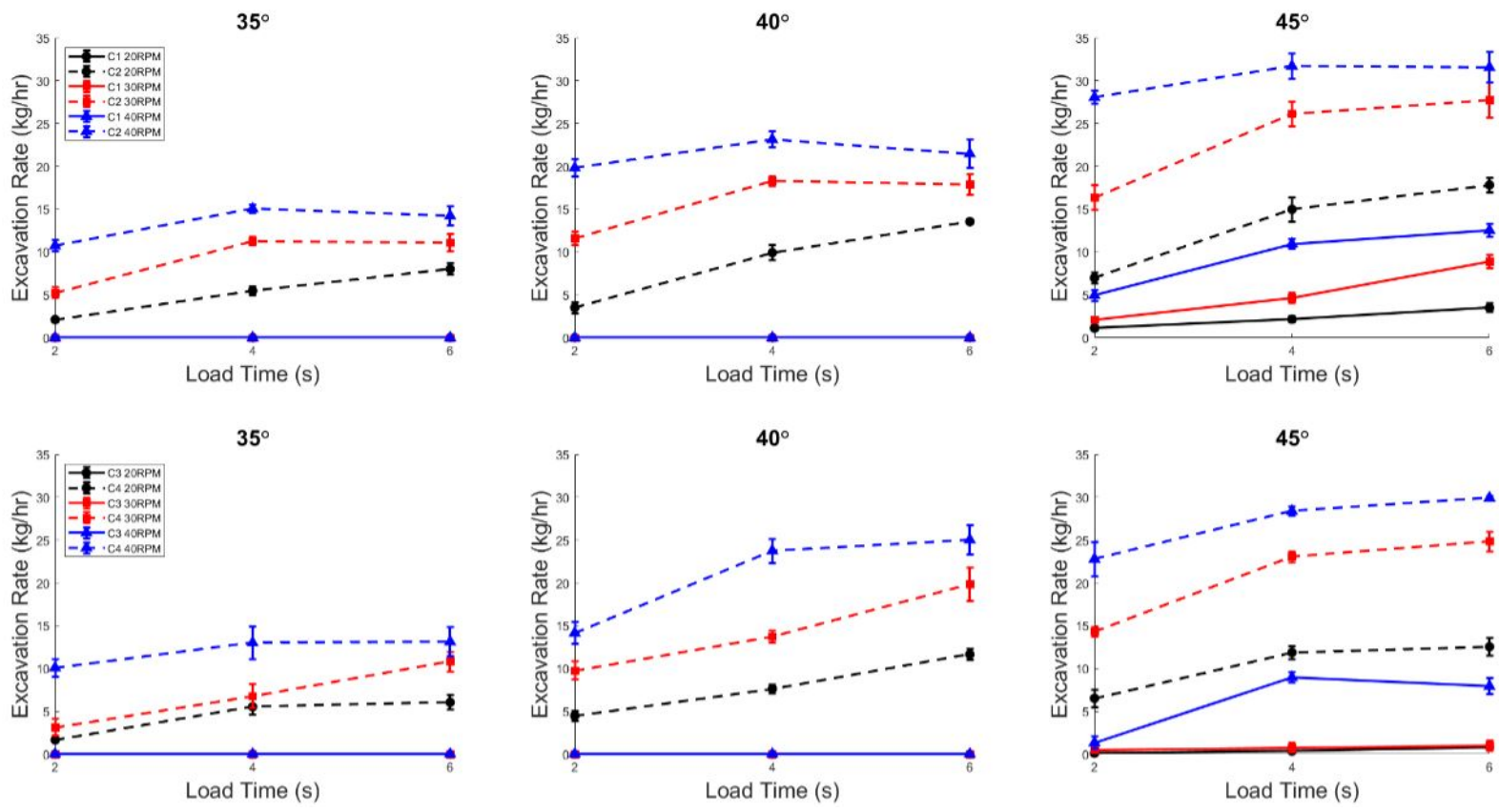

Figure 6: Comparison of average excavation rate $(\mathrm{kg} / \mathrm{hr})$ vs. load time (s) between all configurations for ramp angles of $35^{\circ}, 40^{\circ}$, and $45^{\circ}$, and screw rotational velocities of 20,30 , and $40 \mathrm{RPM}$.

The results in Figure 6 indicate it that the excavation rate behavior of CASPER exhibits quasi-linear trends across all independent variables. For both the wide and narrow outward churning configurations C1 and C3, the ramp system was unable to collect any granular media at ramp angles of 35 and 40 degrees. Even at 45 degrees, C1 and C3 were only able to excavate at 40 RPM, the fastest angular velocity tested. This illustrates that for CASPER, the excavation rate capability can be dramatically increased by simply rotating the front pontoons such that the material is churned inwards, as was done for $\mathrm{C} 2$ and $\mathrm{C} 4$.. In addition, churning inward creates an elevated wake of material, which means the ramp motor requires less angular displacement and thus, less work to deposit the material in the collection bin.

Unsurprisingly, the excavation rate increases as the screw angular velocity increases. This is because there is a greater mass flux of granular media flowing onto the ramp edge, which allows the material to build up to a greater height on the blade itself prior to the ramp rotation. For similar reasons, the excavation rate increases as the load time increases. By increasing the load time, the granular media is given more time to build up prior to ramp rotation. It appears that there is a bit of a diminishing returns phenomenon with 
respect to load time. In other words, the increase from two to four seconds in load time yields a much higher marginal increase in excavation rate in comparison to the marginal increase from four to six seconds. Finally, it was observed that the width between the front pontoons has no significant impact upon the excavation rate.

\section{Power}
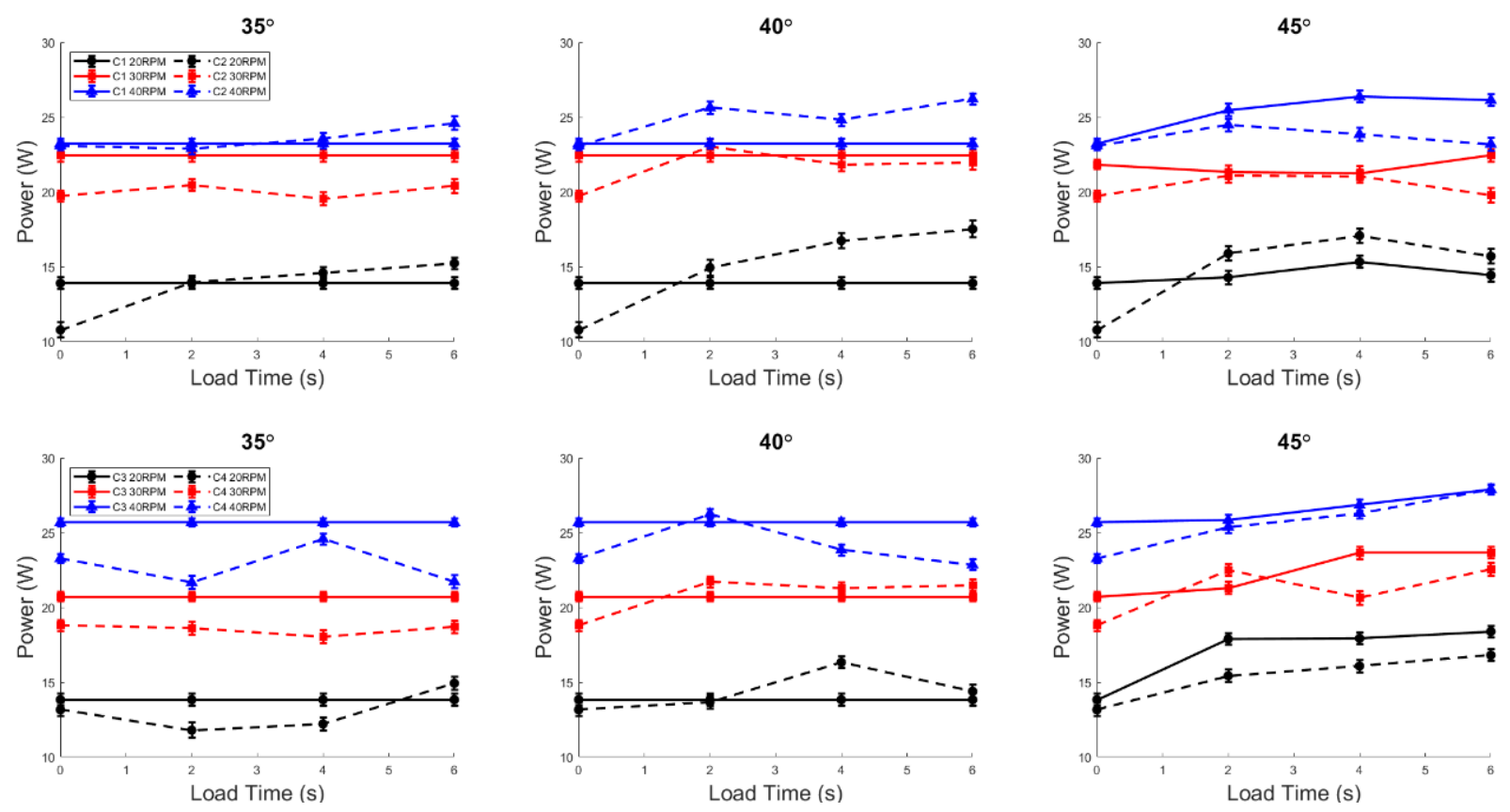

Figure 7: Comparison of average total power (w) vs. load time (s) between all configurations for ramp angles of $0^{\circ}, 35^{\circ}, 40^{\circ}$, and $45^{\circ}$, and screw rotational velocities of 20,30 , and 40 RPM.

Figure 7 illustrates that the total power does increase in a quasi-linear fashion as the screw angular velocities are increased. In most cases, the inward churning configurations $\mathrm{C} 2$ and $\mathrm{C} 4$ exhibited lower power draw than their outward churning counterparts $\mathrm{C} 1$ and $\mathrm{C} 3$. Less intuitively, the power was found to be approximately constant with minor fluctuations with respect to the load time for a given configuration and angular velocity. Initially it was hypothesized that the drag force generated by the ramp-terrain interaction would lead to higher power draw to maintain the velocity, but this was found to not be the case. In all cases where the ramp does not make contact with the soil (C1 and C3 at 35 and 40 degrees), the power draw is nearly identical to the no ramp control case (0 seconds load time). When the ramp does make contact with the soil, the power draw increases slightly above the no ramp level.

It should be noted that the four motors powering the screws consumed the overwhelming majority of the power in all cases. The DC motor actuating the ramp had an astoundingly low power draw of between 0.1-0.2 $\mathrm{W}$, which is one percent of total power. Very low excavation ramp power, coupled with the fact that higher load times do not impose a significant power increase, imply that the most efficient configuration in terms of power per unit excavation rate for this system is the one that maximizes the excavation rate. Furthermore, the supremacy of the inward churning configurations is clear when one considers that the inward churning configurations yield the highest excavation rate, and in most cases draw slightly less power. 


\section{Velocity}
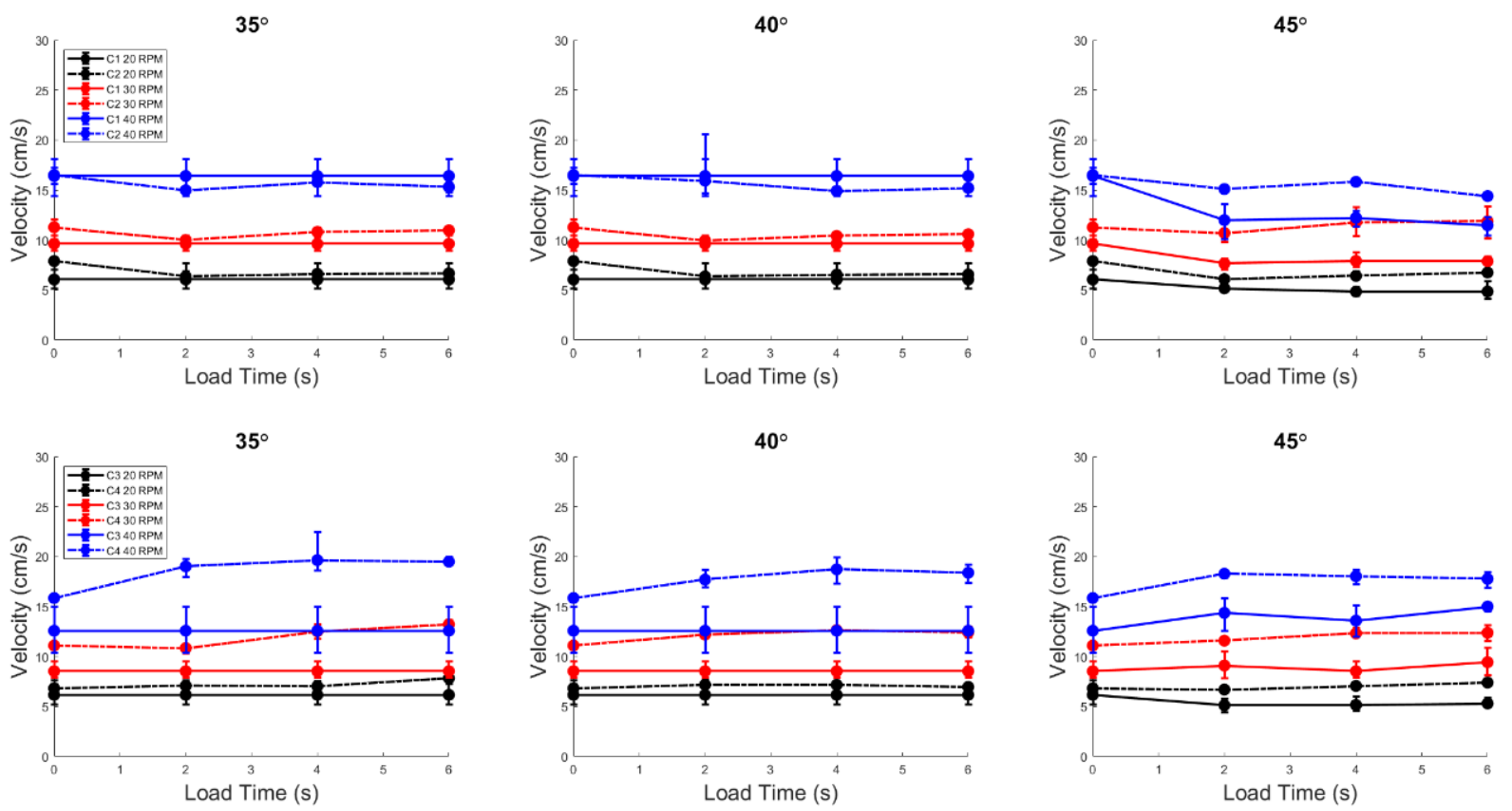

Figure 8: Comparison of average craft velocity vs. load time (s) between all configurations for ramp angles of $0^{\circ}, 35^{\circ}, 40^{\circ}$, and $45^{\circ}$, and screw rotational velocities of 20, 30, and 40 RPM.

Predictably, Figure 8 shows that the velocity increases in a quasi-linear trend as the screw angular velocity increases. This pattern has been demonstrated in the literature (Thoesen et al., 2018, 2019b, 2020e,b) and serves as a sanity check for the velocity results. It is readily apparent that the velocity is fairly constant with respect to load time, as well as ramp angle.

There does not seem to be a significant difference between the wide configurations $\mathrm{C} 1$ and $\mathrm{C} 2$, which implies that when the pontoons are far apart, the material churn direction has negligible impact on the velocity.. However, an interesting disparity exists between the narrow configurations C3 and C4 where the inward churning configuration C3 attains significantly higher velocities than the outward churning C4. This could potentially be explained by how the front pontoon width changes the position of the front pontoon wake. In the wide configurations, the front pontoons are only slightly narrower than the rear pontoons. As a result, the rear pontoons are constantly engaging in media that has already been churned. Conversely, in the narrow configurations, the front pontoons are almost entirely out of the way of the rear pontoons. When churning inward, the rear pontoons are engaging with essentially undisturbed granular media, and thus produce more thrust force than the outward churning case. This effect is likely also present in the wide configuration, but less impact because the front and rear pontoons are nearly in line with each other. Thus, to achieve the highest velocity, the pontoons should be narrow enough such that rear pontoons are engaging undisturbed media and avoiding the front pontoon wake. 

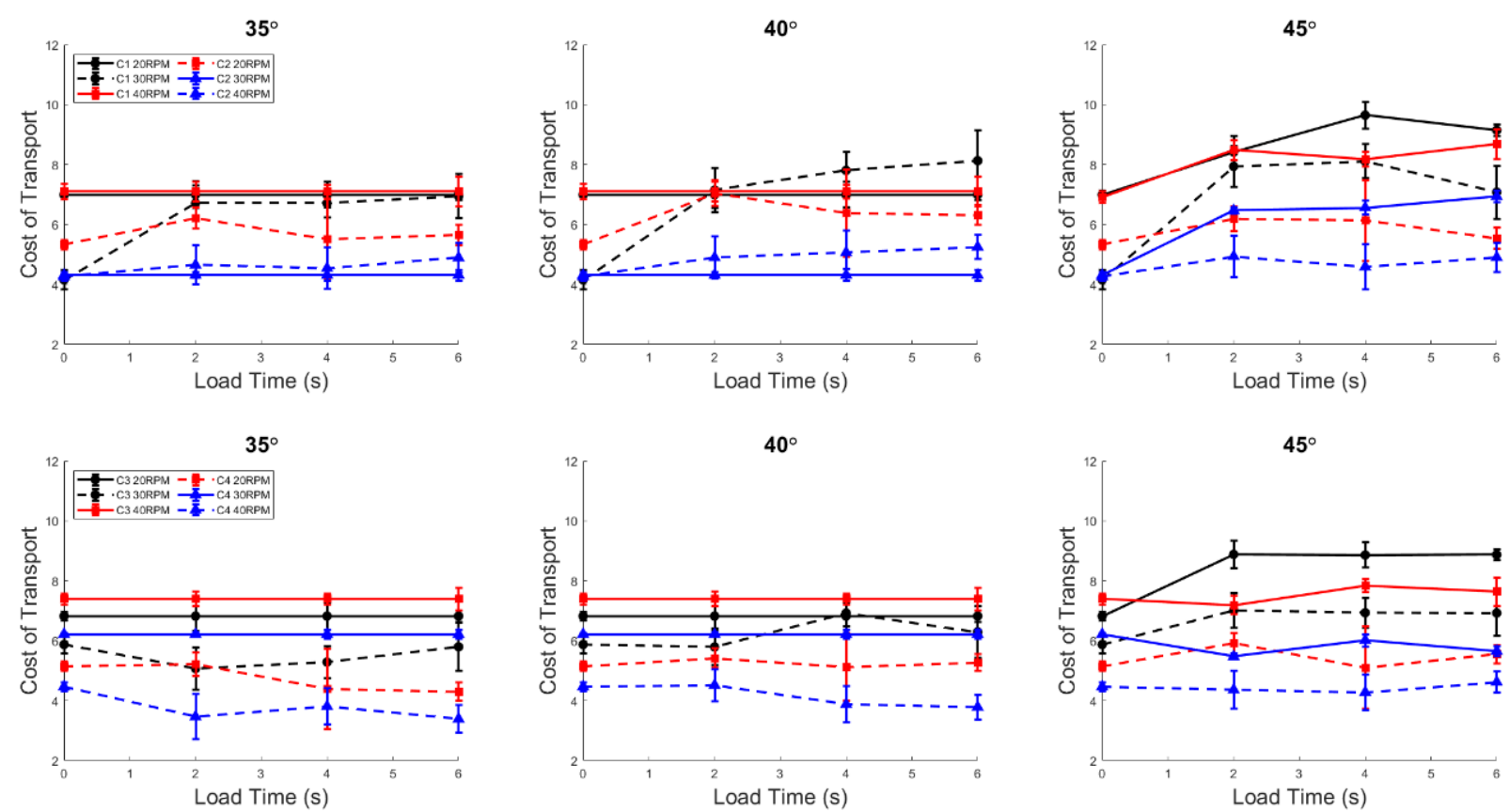

Figure 9: Comparison of average cost of transport vs. load time (s) between all configurations for ramp angles of $0^{\circ}, 35^{\circ}, 40^{\circ}$, and $45^{\circ}$, and screw rotational velocities of 20,30 , and 40 RPM.

\section{Cost of Transport}

The cost of transport (COT) is a highly informative parameter that characterizes the mobility performance of the vehicle, and is especially useful when comparing one vehicle to another. Therefore, the COT distribution of CASPER was calculated so that the optimal settings for mobility performance could be identified. The cost of transport is defined in Equation ?? below, where $P, W_{c}$, and $V$ are the craft power, weight, and velocity, respectively.

$$
C O T=\frac{P}{W_{c} \cdot V} C O T=\frac{P}{W_{c} \cdot V}
$$

Generally, Figure 9 shows that the cost of transport reduces as the RPM increases, and that the COT is not significantly impacted by load time. Comparing the top and bottom rows of Figure 9, it is clear that the narrow/wide configurations have roughly the same cost of transport at all angular velocities and load times. From these graphs, it can be concluded that cost of transport is minimized (or that the mobility system is most efficient) when the screws are operating at high angular velocity. This implies that they move the fastest for a given amount of power, and thus are more efficient for mobility in granular media. In the next section, the cost of transport and excavation rate will be investigated simultaneously to quantify the mobility and excavation performance as a whole. Conveniently, the excavation system is also most effective 
when operating at high screw angular velocities. It is critical for a screw-propelled excavation to operate in the configuration that minimizes the cost of transport, because screws present an inherent disadvantage to wheels and tracks when travelling in the counter-rotating mode during the excavation process.

\section{Excavation Transport Rate}
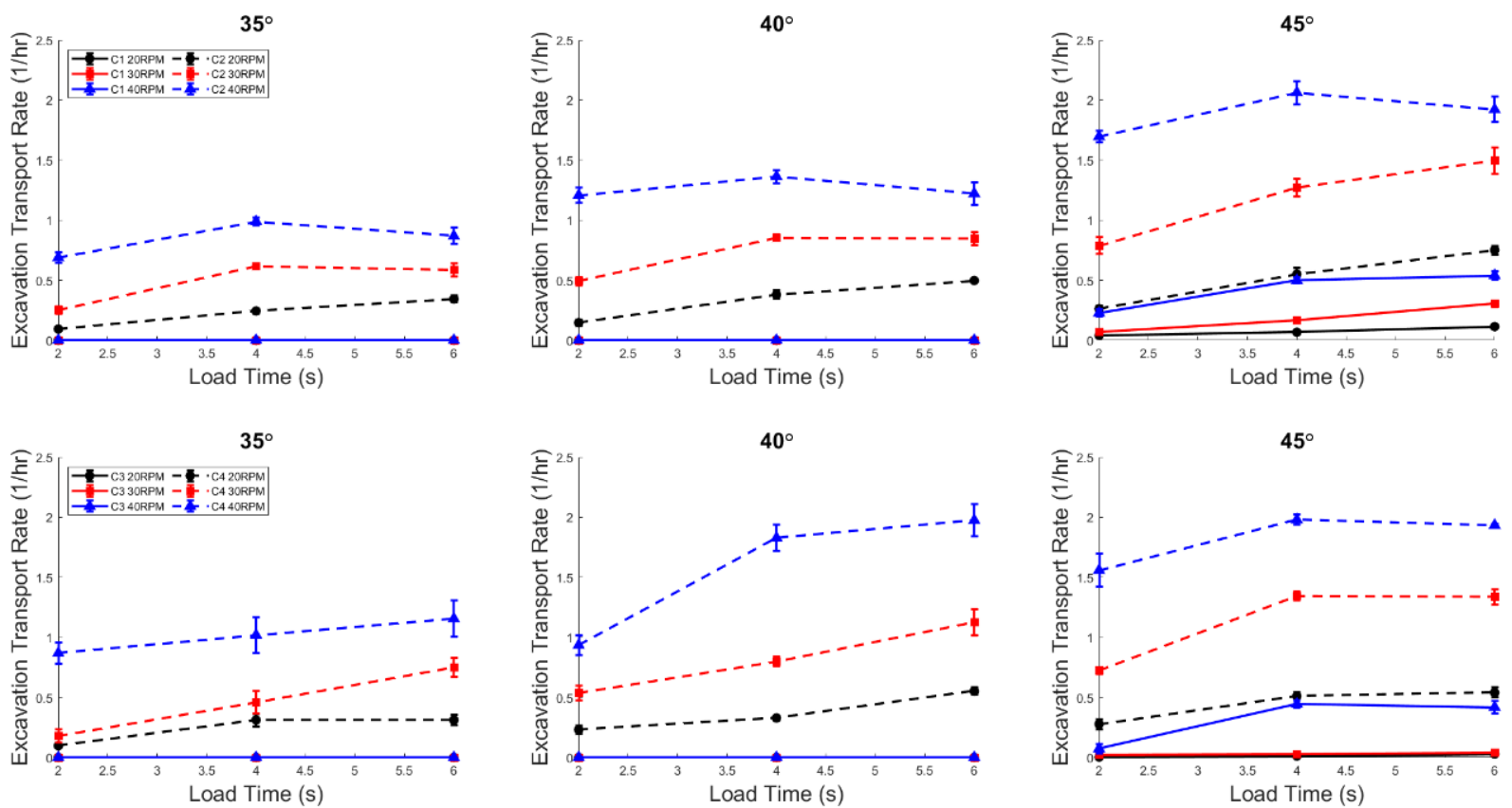

Figure 10: Comparison of average excavation rate/cost of transport vs. load time (s) between all configurations for ramp angles of $35^{\circ}, 40^{\circ}$, and $45^{\circ}$, and screw rotational velocities of 20,30 , and 40 RPM

The excavation transport rate (ETR) is a new parameter we introduce that can represent the overall transportation and excavation efficiency of a hybrid mobility-excavation system. ETR is defined in Equation ??, where $R_{E}$ is the excavation rate, $m_{c}$ is the craft mass, and COT is the cost of transport.

$E T R=\frac{R_{E}}{m_{c} \cdot C O T}$

$(2)$

It should be noted that a higher value of ETR provides higher performance because more mass is being collected at a lower cost of transport. Figure 10 shows a clear trend that is consistent across all configurations, angular velocities, and load times. The ETR increases in a quasi-linear fashion with load time. Interestingly, as the load time increases, the ETR appears to asymptotically approach a maximum value. A similar trend exists between ETR and angular velocity. Increasing the angular velocity from 20 to 30 RPM has a significantly larger increase in ETR than increasing it from 30 to 40 RPM in all cases. There does not appear to be a significant difference between the wide and narrow configurations $\mathrm{C} 2$ and $\mathrm{C} 4$, as 
both of these follow very similar trends and end up approaching the same maximum ETR values at each given ramp angle.

Note that only the 45 degree cases have nonzero values for $\mathrm{C} 1$ and $\mathrm{C} 3$ because they were the only ones that had nonzero excavation rates. When comparing the inward/outward churning configurations, it is obvious that the inward churning configurations have significantly higher ETR values. This dramatic difference is illustrative of the fact that the inward churning configurations are more efficient for excavation than their outward churning counterparts while maintaining a similar cost of transport. Additionally, it is evident that increasing the ramp angle, load time, and RPM all lead to the most efficient configurations. Furthermore, for both of the outward churning configurations at the 40 and 45 degrees ramp angles, there does not appear to be a significant difference in ETR between the four and six second load time cases at 30 and 40 RPM. These would appear to be the most efficient configuration of all settings. In a space environment, one might opt for 30 RPM to be at peak efficiency with lower total power required. The lower angular velocity would provide a lower total power requirement, which in turn would lower the mass of the spacecraft. For terrestrial applications, it would be likely to favor the faster angular velocity which would maximize the excavation rate. Ultimately it appears that there is an optimum ETR value that can be achieved with many different configurations of load time, ramp angle, and angular velocity.

In general, it was found that the inward churning configurations C2 and C4 were able to collect significantly more mass at practically the same total power while traveling at the same or greater speeds in comparison to their outward churning counterparts $\mathrm{C} 1$ and $\mathrm{C} 3$ across all load times, ramp angles, and angular velocities.

It was observed that the inward churning configurations created an elevated wake behind the front screws, directly in the path of the ramp. Conversely, the outward churning configurations diverted the sand away from the ramp, leaving a strip of undisturbed flat sand in front of the ramp. A detailed image of the wake patterns for each configuration is shown in Figure 4. It is noteworthy that the length of the depression cut by the ramp is directly proportional to the independent variable, load time. Additionally, since the outward churning configurations did not have the elevated wake, the ramp angle needed to be 45 degrees to just barely make contact with the soil. As a result, $\mathrm{C} 1$ and $\mathrm{C} 3$ were only able to collect mass at the 45 degree ramp angle. Attempts were made to collect material at 35 and 40 degrees, but were unsuccessful. This phenomenon makes it evident that having the front screws churn material inward provided a significant benefit to act as an auxiliary mechanism for excavation.

In order to compare CASPER to other ISRU excavation systems, a table of characteristic values is presented in Table 1. Note that the presented values correspond to CASPER's optimal configuration of C2, 40 RPM, 45 degree ramp angle, and 4 second load time. A comprehensive performance comparison between many ISRU excavators was performed by Just et al. (Just et al., 2020a) and the metrics reported in Table 1 are the same as those used in that comparison.

Tabelle 1: CASPER Performance Metrics

\begin{tabular}{cccccc}
\hline Excavation rate & Depth of cut & System mass & Power requirement & Traverse speed & Simulant used \\
\hline $30 \mathrm{~kg} / \mathrm{hr}$ & $2.5 \mathrm{~cm}$ & $3.4 \mathrm{~kg}$ & $<30 \mathrm{~W}$ & $10.2 \mathrm{~m} / \mathrm{min}$ & Sand \\
\hline
\end{tabular}

CASPER yields a lower excavation rate than other discrete excavators, such as the Backhoe (100 kg/ $\mathrm{hr}$ (van Susante and Dreyer, 2010)) and Cratos (900kg/hr (Greer et al., 2013)) systems. However, CASPER delivers a serviceable excavation rate at a much lower system mass $(\sim 80 \mathrm{~kg}$ for both Backhoe and Cratos) and much lower power requirements ( $\sim 200 \mathrm{~W}$ for Backhoe and $\sim 100 \mathrm{~W}$ for Cratos) while traveling at similar speeds. Furthermore, CASPER yields a nearly identical ETR to the Backhoe system. Based on the available power, excavation rate, system mass, and velocity data (van Susante and Dreyer, 2010; Just et al., 2020a), the Backhoe system has an ETR of 2.01. This is remarkably close to CASPER's maximum ETR of 1.97-2.06. From these comparisons, it can be concluded that although CASPER delivers lower excavation rates than other discrete excavation systems, it does so with a much lower system mass and lower power requirements. In addition, the novel ETR parameter makes it clear that CASPER provides performance as a mobile excavator 
as larger systems in a much smaller package. This implies that CASPER might be better suited for extraterrestrial applications where power and mass must be minimized. Moreover, for applications where higher excavation rates are needed, CASPER can be scaled up to accommodate those needs. However, further studies are warranted to extend current helical granular scaling laws to excavation systems (Thoesen et al., 2020d).

\section{Conclusion}

The purpose of this investigation was to characterize the excavation and mobility performance of the CASPER robot, a novel discrete scooper hybrid excavation platform. CASPER is propelled by four counter rotating Archimedes screws. These screws serve as both the mobility system, as well as augmenting the excavation system with the front screws churning up the granular media in order to be discretely scooped into the collection bin via the ramp mechanism. Four major configurations of CASPER were investigated, consisting of narrow and wide variants of inward and outward churning front screws.

The major performance parameters measured included excavation rate, power consumption, velocity, cost of transport, and a new parameter, the excavation transport rate. These dependent variables were measured over a range of the independent variables of screw angular velocity, ramp load time, ramp angle, and front screw configuration. It was found that CASPER had a peak excavation rate of around $30 \mathrm{~kg} / \mathrm{hr}$ at the optimal settings of 40 RPM and 4 seconds of load time. The operational power of CASPER ranged from approximately $10-15 \mathrm{~W}$ for $20 \mathrm{RPM}$ cases up to $20-25 \mathrm{~W}$ for the $40 \mathrm{RPM}$ cases and exhibited very little variation with respect to load time. The velocity performance exhibited similar trends to the power consumption, with velocities ranging from $5-18 \mathrm{~cm} / \mathrm{s}$ for $20-40 \mathrm{RPM}$ with very little variation with load time. The cost of transport results indicated that higher angular velocities are more efficient for mobility, insofar as they move quicker for a given amount of power. It is important to recognize that these velocity and power data are highly specific to the Quikrete silica sand that the craft was tested in. In planetary regolith analogues, which have smaller, cohesive, and more angular particles, the power consumption would be expected to increase, and the velocity would be expected to decrease (Thoesen et al., 2020e). In other words, the harsher geological properties of regolith found on planetary bodies will have an adverse impact on the mobility performance. The most critical parameter investigated, the excavation transport rate, aims to quantify a mixture of mobility and excavation performance that is normalized by the craft mass to enable clearer comparison between existing systems. The ETR results indicated that the optimal configuration of CASPER was a 45 degree ramp angle, 40 RPM angular velocity, with a load time of 4 seconds with the wide inward churning configuration of the front screw pontoons. This configuration had the best combination of excavation rate, power consumption, and velocity. The outward churning configurations were ineffective due to the fact that they did not create the raised section of pre-churned material in front of the ramp that the inward churning configurations did. According to the ETR results, the narrow and wide configurations were very similar, with the narrow configuration having a slight advantage at lower ramp angles. This is likely due to the height of the elevated wake behind the front pontoons being slightly higher from the close proximity of the pontoons, which allows the ramp to make contact at a lower angle.

Some promising avenues of future work have been identified during this investigation. First and foremost, increasing the scale of CASPER and operating it in a field environment would be key in determining if this architecture would be suitable for extra-terrestrial ISRU and terrestrial mining applications. One major avenue of upgrading CASPER's capability would be adding a navigation system in order to precisely control the direction of travel and correct for any deviations due to the lateral forces induced by the excavation ramp. Furthermore, adding a system to measure the excavated mass in real time would enable an investigation on how the dynamics and mobility performance of the craft is impacted as it takes on mass. Ultimately, this investigation has shown a great deal of promise for discrete scooper excavation systems, and has shown that Archimedes screw propulsion systems can substantially augment the excavation capabilities as long as the screws rotate in the proper direction to churn material towards the excavation system. 


\section{Acknowledgements}

The authors would like to thank the members of the ASU BIRTH Lab for their assistance and Arizona State University for funding.

\section{Conflict of interest}

The authors report no conflicts of interest.

\section{References}

Haym Benaroya, Leonhard Bernold, and Koon Meng Chua. Engineering Design and Construction of Lunar Bases. Journal of Aerospace Engineering, 15(2):33-45, apr 2002. doi: 10.1061/(asce)0893-1321(2002)15: 2(33). URL https://doi.org/10.1061\%2F\%28asce\%290893-1321\%282002\%2915\%3A2\%2833\%29.

Brad R Blair, Javier Diaz, Michael Duke, Elisabeth Lamassoure, Robert Easter, Mark Oderman, and Marc Vaucher. Space resource economic analysis toolkit: The case for commercial lunar ice mining. Final report to the NASA Exploration Team, 2002.

John Caruso, Lawrence Greer, John Wentworth, Spina Dan, Mike Krasowski, Phillip Abel, Norman Prokop, Joseph Flatico, and Kurt Sacksteder. Cratos: A Simple Low Power Excavation and Hauling System for Lunar Oxygen Production and General Excavation Tasks. In PTMSS Conference, 2013.

Giovanni Cesaretti, Enrico Dini, Xavier De Kestelier, Valentina Colla, and Laurent Pambaguian. Building components for an outpost on the Lunar soil by means of a novel 3D printing technology. Acta Astronautica, 93:430-450, jan 2014. doi: 10.1016/j.actaastro.2013.07.034. URL https://doi.org/10.1016\%2Fj . actaastro.2013.07.034.

Ian A Crawford. Lunar resources: A review. Progress in Physical Geography, 39(2):137-167, 2015.

Ken Evans. The History Challenges, and New Developments in the Management and Use of Bauxite Residue. Journal of Sustainable Metallurgy, 2(4):316-331, may 2016. doi: 10.1007/s40831-016-0060-x. URL https: //doi.org/10.1007\%2Fs40831-016-0060-x.

Lawrence C Greer, Michael J Krasowski, Norman F Prokop, and Dan C Spina. Cratos: The Evolution of a Robotic Vehicle. Technical report, NASA/TM-2013-216491, 2013.

Matt Heverly, Jaret Matthews, Justin Lin, Dan Fuller, Mark Maimone, Jeffrey Biesiadecki, and John Leichty. Traverse Performance Characterization for the Mars Science Laboratory Rover. Journal of Field Robotics, 30(6):835-846, sep 2013. doi: 10.1002/rob.21481. URL https://doi.org/10.1002\%2Frob. 21481.

Christopher A Jones, Jordan Klovstad, Emily Judd, and David Komar. Cost breakeven analysis of cis-lunar ISRU for propellant. In AIAA Scitech 2019 Forum, page 1372, 2019.

Liang Ju, Gabriele Ferri, Cecilia Laschi, Barbara Mazzolai, and Paolo Dario. Experimental results of a novel amphibian solution for aquatic robot. In 2010 IEEE International Conference on Robotics and Automation. IEEE, may 2010. doi: 10.1109/robot.2010.5509773. URL https://doi.org/10.1109\%2Frobot. 2010. 5509773. 
G.H. Just, K. Smith, K.H. Joy, and M.J. Roy. Parametric review of existing regolith excavation techniques for lunar In Situ Resource Utilisation (ISRU) and recommendations for future excavation experiments. Planetary and Space Science, 180:104746, jan 2020a. doi: 10.1016/j.pss.2019.104746. URL https://doi. org/10.1016\%2Fj.pss. 2019.104746.

GH Just, K Smith, KH Joy, and MJ Roy. Parametric review of existing regolith excavation techniques for lunar In Situ Resource Utilisation (ISRU) and recommendations for future excavation experiments. Planetary and Space Science, 180:104746, 2020b.

N. Labeaga-Martínez, M. Sanjurjo-Rivo, J. Díaz-Álvarez, and J. Martínez-Frías. Additive manufacturing for a Moon village. Procedia Manufacturing, 13:794-801, 2017. doi: 10.1016/j.promfg.2017.09.186. URL https://doi.org/10.1016\%2Fj.promfg.2017.09.186.

Tony Lavoie and Paul D Spudis. The purpose of human spaceflight and a lunar architecture to explore the potential of resource utilization. In AIAA SPACE 2016 Conference and Exposition, AIAA SPACE Forum,(AIAA 2016-5526), 2016.

Shuai Li, Paul G Lucey, Ralph E Milliken, Paul O Hayne, Elizabeth Fisher, Jean-Pierre Williams, Dana M Hurley, and Richard C Elphic. Direct evidence of surface exposed water ice in the lunar polar regions. Proceedings of the National Academy of Sciences, 115(36):8907-8912, 2018.

Robert P Mueller and Robert H King. Trade study of excavation tools and equipment for lunar outpost development and ISRU. In AIP conference proceedings, volume 969, pages 237-244. American Institute of Physics, 2008.

Robert P Mueller, Rachel E Cox, Tom Ebert, Jonathan D Smith, Jason M Schuler, and Andrew J Nick. Regolith advanced surface systems operations robot (RASSOR). In 2013 IEEE Aerospace Conference, pages 1-12. IEEE, 2013.

V. Nardelli, M.R. Coop, J.E. Andrade, and F. Paccagnella. An experimental investigation of the micromechanics of Eglin sand. Powder Technology, 312:166-174, may 2017. doi: 10.1016/j.powtec.2017.02.009. URL https://doi.org/10.1016\%2Fj.powtec. 2017.02.009.

M.J. Neumeyer and B.D. Jones. The Marsh Screw Amphibian. Journal of Terramechanics, 2(4):83-88, jan 1965. doi: 10.1016/0022-4898(65)90133-3. URL https://doi.org/10.1016\%2F0022-4898\%2865\% 2990133-3.

Gerald Sanders. Comparison of lunar and Mars in-situ resource utilization for future robotic and human missions. In 49th AIAA Aerospace Sciences Meeting Including the New Horizons Forum and Aerospace Exposition, page 120, 2011.

Gerald B Sanders and William E Larson. Progress made in lunar in situ resource utilization under NASA's exploration technology and development program. Earth and Space 2012: Engineering, Science, Construction, and Operations in Challenging Environments, pages 457-478, 2012.

Prabal Saxena, Rosemary M Killen, Vladimir Airapetian, Noah E Petro, Natalie M Curran, and Avi M Mandell. Was the Sun a slow rotator? Sodium and potassium constraints from the lunar regolith. The Astrophysical Journal Letters, 876(1):L16, 2019.

Krzysztof Skonieczny, Scott J Moreland, David Wettergreen, and William Whittaker. Advantageous bucketwheel configuration for lightweight planetary excavators. 2011.

Krzysztof Skonieczny, DS Wettergreen, and WL "Red" Whittaker. Advantages of continuous excavation in lightweight planetary robotic operations. The International Journal of Robotics Research, 35(9):1121-1139, 2016.

Paul Spudis and Anthony Lavoie. Using the resources of the Moon to create a permanent, cislunar space fairing system. In AIAA Space 2011 Conference \& Exposition, page 7185, 2011. 
Andrew Thoesen, Sierra Ramirez, and Hamid Marvi. Screw-powered propulsion in granular media: An experimental and computational study. In 2018 IEEE International Conference on Robotics and Automation (ICRA), pages 4283-4288. IEEE, 2018.

Andrew Thoesen, Teresa McBryan, and Hamidreza Marvi. Helically-driven granular mobility and gravityvariant scaling relations. RSC Advances, 9(22):12572-12579, 2019a. doi: 10.1039/c9ra00399a. URL https://doi.org/10.1039\%2Fc9ra00399a.

Andrew Thoesen, Teresa McBryan, and Hamidreza Marvi. Helically-driven granular mobility and gravityvariant scaling relations. RSC advances, 9(22):12572-12579, 2019b.

Andrew Thoesen, Sierra Ramirez, and Hamid Marvi. Screw-generated forces in granular media: Experimental, computational, and analytical comparison. AIChE Journal, 65(3):894-903, 2019c.

Andrew Thoesen, Teresa McBryan, Marko Green, Darwin Mick, Justin Martia, and Hamid Marvi. Revisiting Scaling Laws for Robotic Mobility in Granular Media. IEEE Robotics and Automation Letters, 5(2):13191325, apr 2020a. doi: 10.1109/lra.2020.2968031. URL https://doi.org/10.1109\%2Flra.2020. 2968031.

Andrew Thoesen, Teresa McBryan, Marko Green, Darwin Mick, Justin Martia, and Hamid Marvi. Revisiting scaling laws for robotic mobility in granular media. IEEE Robotics and Automation Letters, 5(2):13191325, 2020b.

Andrew Thoesen, Teresa McBryan, Darwin Mick, Marko Green, Justin Martia, and Hamid Marvi. Comparative performance of granular scaling laws for lightweight grouser wheels in sand and lunar simulant. Powder Technology, 373:336-346, aug 2020c. doi: 10.1016/j.powtec.2020.05.114. URL https: //doi.org/10.1016\%2Fj. powtec. 2020.05.114.

Andrew Thoesen, Teresa McBryan, Darwin Mick, Marko Green, Justin Martia, and Hamid Marvi. Granular scaling laws for helically driven dynamics. Physical Review E, 102(3), sep 2020d. doi: 10.1103/physreve. 102.032902. URL https://doi.org/10.1103\%2Fphysreve.102.032902.

Andrew Thoesen, Teresa McBryan, Darwin Mick, Marko Green, Justin Martia, and Hamid Marvi. Comparative performance of granular scaling laws for lightweight grouser wheels in sand and lunar simulant. Powder Technology, 373:336-346, 2020e.

Paul J. van Susante and Chris B. Dreyer. Lunar and Planetary Excavation Prototype Development and Testing at the Colorado School of Mines. In Earth and Space 2010. American Society of Civil Engineers, mar 2010. doi: 10.1061/41096(366)109. URL https://doi.org/10.1061\%2F41096\%28366\%29109.

David Wettergreen, Dominic Jonak, David Kohanbash, Scott Moreland, Spencer Spiker, James Teza, and William Whittaker. Design and experimentation of a rover concept for lunar crater resource survey. In 47th AIAA Aerospace Sciences Meeting Including The New Horizons Forum and Aerospace Exposition, page 1206, 2009.

David Wettergreen, Scott Moreland, Krzysztof Skonieczny, Dominic Jonak, David Kohanbash, and James Teza. Design and field experimentation of a prototype lunar prospector. The International Journal of Robotics Research, 29(12):1550-1564, 2010.

Allen Wilkinson and Alfred DeGennaro. Digging and pushing lunar regolith: Classical soil mechanics and the forces needed for excavation and traction. Journal of Terramechanics, 44(2):133-152, apr 2007. doi: 10.1016/j.jterra.2006.09.001. URL https://doi.org/10.1016\%2Fj .jterra.2006.09.001.

Xiangwu Zeng, Louis Burnoski, Juan Agui, and Allen Wilkinson. Calculation of Excavation Force for ISRU on Lunar Surface. In 45th AIAA Aerospace Sciences Meeting and Exhibit. American Institute of Aeronautics and Astronautics, jan 2007. doi: 10.2514/6.2007-1474. URL https://doi.org/10.2514\%2F6.2007-1474.

Anthony Zuppero, Bruce Schnitzler, Thomas Larson, Anthony Zuppero, Bruce Schnitzler, and Thomas 
Larson. Nuclear-heated steam rocket using lunar ice. In 33rd Joint Propulsion Conference and Exhibit, page 3172, 1997. 